"If you listen to me properly, I feel good": A qualitative examination of patient experiences of dietetic consultations

Running headline: Patient experiences of dietetic consultations

Six key words: Behaviour change; consultations; dietitians; patient-centred; patientperception; patient perspective; patient preferences.

Authors: Ruth E.E. Hancock ${ }^{1,2}$, Gillie Bonner ${ }^{3}$, Rachel Hollingdale ${ }^{4}$, Angela M. Madden $^{1}$

Work undertaken in the School of Human Sciences, London Metropolitan University, London N7 8DB; School of Health and Emergency Professions, University of Hertfordshire, Hatfield AL10 9AB; and Division of Nutritional Sciences, University of Surrey, Guildford GU2 7TE.

Present addresses:

${ }^{1}$ School of Health and Emergency Professions, University of Hertfordshire, Hatfield, AL10 9AB, UK

${ }^{2}$ Nutrition and Dietetics, University Hospitals Bristol NHS Foundation Trust, Bristol, BS2 8HW, UK

${ }^{3}$ Michael Rutter Centre, South London and Maudsley NHS Foundation Trust, London, SE5 8AZ, UK

${ }^{4}$ Brighton and Hove Food Partnership, Brighton, BN41 2PA, UK. 
Corresponding author: $\operatorname{Dr}$ Angela Madden, School of Health and Emergency Professions, University of Hertfordshire, Hatfield AL10 9AB; telephone 01707 281385; fax 01707 284977; email a.madden@herts.ac.uk

Roles of authors: REEH designed and carried out the data analysis, undertook a literature review and wrote the first draft of the manuscript; GB conceived the original study idea, designed the study, undertook all data collection and contributed to all other aspects; $\mathrm{RH}$ contributed to the design of data analysis, carried out data analysis and contributed to the drafting of the manuscript; AMM contributed to the overall study design, supervised the study throughout and completed the manuscript. All authors critically reviewed the manuscript and approved the final version submitted for publication.

Conflict of interests: None

Funding: The Health Foundation provided financial support for part of the study through a 'Leading Practice Through Research Award' made to Gillie Bonner. 


\title{
"If you listen to me properly, I feel good": A qualitative examination of patient experiences of dietetic consultations
}

\begin{abstract}
Background: There is considerable interest in healthcare research regarding communication skills and some debate surrounding the effectiveness of a patientcentred approach to care. Understanding patient experiences of consultations can help indicate how consultations can be modified to improve effectiveness. At present, there is little research exploring patient experience of dietetic consultations. The aim of this study was to achieve a better understanding of patients' experiences of dietetic consultations using qualitative analysis.
\end{abstract}

Methods: Patients undergoing consultations with a dietitian were invited to discuss their experience of the consultation with a research dietitian not involved in their care. Individual interviews and focus groups were conducted and analyzed using the Framework approach.

\section{Results:}

Seventeen patients participated and described experiences of consultations which were varied and influenced by factors such information given (resources, explanation, repetition, consistency), their dietitian's approach (prescriptive or nonprescriptive, use of behaviours change skills) and behaviour (listening skills, body language) and appointment (expectations, involvement of the multidisciplinary team, length of time) as well as their own internal experience (confidence, guilt, frustration). Patients agreed that certain factors, such as good communication and rapport, receiving effective and reliable information and resources, and non-judgmental, regular support were important factors in creating a positive experience of their consultation. However, they differed in what they believed constituted these factors. 
Conclusions: Patients like dietitians to adopt a patient-centred approach, which might be either patient- or practitioner-led, and to take account of what they wanted from consultations, adapting these to meet their individual requirements. 


\section{Introduction}

There is consensus that nutritional intake plays a role both in optimizing health and in the pathogenesis of long term conditions including obesity, diabetes, cardiovascular disease and certain cancers (Boffetta et al., 2010; Carter et al., 2010; Goodpaster et al., 2010). Debate often focuses on what dietary changes are required to maximise health benefits but less attention has been given to the approach taken when counselling to facilitate dietary change or the way this information is delivered or patients' preference. In terms of wider healthcare delivery and decision making, there has been a shift towards a 'patient-centred' approach which involves patients at all stages (World Health Organisation, 2000; Department of Health, 2010). The term 'patient-centred' has a broad range of interpretation (Lewin et al., 2001), but is usually presented as an alternative to the traditional medical model of ill health, which focuses on the health practitioner as 'expert' (Curry \& Jaffe, 1998). Mead and Bower (2000) identify five common components of the patient-centred approach: (i) a bio-psychosocial perspective; (ii) an effort to understand the patients' experience of their illness; (iii) a sharing of responsibility and power between the patient and clinician; (iv) a therapeutic alliance between the two; and (v) the importance of the personal relationship built between the patient and clinician.

Within the research surrounding the patient-centred approach, there is an increasing body of evidence supporting the importance of communication skills (Najavits \& Weiss, 1994). Patients have not always been satisfied with the level of communication with their healthcare professionals (Audit Commission, 1993), and it is suggested that an improvement in communication between patients and 
healthcare professionals allows an intervention to become more focused on the needs of the patient (Stewart \& Browne, 1996). Within dietetics, communication skills are explicitly included in professional standards (Dietitians of Canada, 1996; O'Sullivan-Maillet et al., 2005; Health Professions Council, 2007) and are highly valued by practitioners (Whitehead et al., 2009). A patient-centred, counselling approach is seen as an essential element of dietetic practice (Gable, 2007), although there is not universal understanding of the concept amongst dietitians (MacLellan \& Berenbaum, 2006). In addition, although there is evidence that dietitians perceive themselves to be effective in using counselling skills, this has not yet been related to their actual counselling performance (Lu \& Dollahite, 2010).

Studies have looked specifically at dietitians' verbal and non-verbal communication skills, identifying key areas of communication and concluding that non-verbal aspects of communication have significant impact on the effectiveness of consultations (Cant \& Aroni, 2008; Cant, 2009). Goodchild et al., (2005), investigated empathy in dietetic consultations, and concluded that patients whose dietitian scored more highly on a prescribed scale were more satisfied with the outcome of their consultations. Jones et al., (2007), investigated obese adults' opinions of the outcome of dietetic intervention and noted that patients identified the support of the dietitian as important in achieving weight loss. Most research has tended to focus on the outcome of intervention rather than the patient experience or the dietitian-patient relationship (Cant \& Aroni, 2008), although some studies have used a questionnaire scale to assess patient satisfaction with treatment and concluded that interpersonal skills and personal presentation impacted on patient satisfaction (Ferguson et al., 2001; Vivanti et al., 2007). Hollingdale et al., (2008), 
used a questionnaire developed through patient focus groups to assess the experiences of renal patients receiving dietetic advice, but there is little other work examining the experiences of dietetic patients. Clearly this is needed if dietitians are to be effective facilitators of the dietary change that has the potential to yield considerable health benefits.

The aim of this investigation was, therefore, to explore qualitatively patients' experiences of dietetic consultations in order to achieve a better understanding of their perspectives. 


\section{Methods}

Approval for the study was given by the NHS Research Ethics Committee (04/Q1604/121) and the Ethics Committee of London Metropolitan University.

\section{Participants and recruitment}

The present study was part of a larger investigation (Bonner et al., 2008) which had three recruitment stages. (1) Dietitians working in three NHS Trusts located in two cities in the south of the UK were invited to participate in the study. These included community- and hospital-based staff working in both general and specialist roles and with a variation in background and experience. Thirty-three dietitians consented to participate. (2) The participating dietitians then invited patients who were attending for routine outpatient dietetic appointments to take part in the study by allowing their consultation to be audio- recorded and then, later, to take part in an interview or focus group. The inclusion criteria were patients aged 18 years or more who could speak English and were attending for a consultation relating to a long-term condition, e.g. obesity, diabetes or cardiovascular disease. Forty-one patients consented to participate. (3) A research dietitian (GB) who was not involved with patient care in any of the three NHS Trusts invited the patients to attend either individual semistructured interviews or a focus group where their experience of their dietetic consultation was discussed. Eleven patients consented and participated in the interviews and a further six patients consented and participated in two focus groups.

\section{Data collection}

Individual interviews - Eleven individual semi-structured interviews lasting approximately 30-60 minutes were held in meeting or consultation rooms in the 
hospital where the patients' consultations had taken place. The interviews were arranged for different times of the day and week that were mutually convenient for the participants and interviewer. The research dietitian (GB) acted as interviewer for all sessions. The topic guide (Appendix 1) was used to ensure the relevant areas were addressed and provide consistency between interviews although participants were also allowed to talk about issues that were important to them but not included in the guide. Each interview began by providing opportunities for the participants to (i) express their feelings about their consultation and (ii) describe the overall impact on their eating behaviour. It then continued by reviewing what the participants reported the dietitians said or did that resulted in the patients feeling (i) (dis)satisfied with the consultation, (ii) (un)motivated to make/sustain changes to their diet, (iii) (un)confident about making/sustaining changes to their diet, (iv) feelings of being accepted and understood. The discussion was audio-recorded by two unobtrusive tape recorders.

Focus groups - One focus group was held in each of the two cities where the participating Trusts were located and took place in the evening for convenience of the participants. Each lasted approximately $1 \frac{1 / 2}{2}$ hours and refreshments were provided. The focus group was moderated by the research dietitian (GB). The participants sat around a table in a room set up to provide a relaxed but practical environment and were free to take refreshments during the session. At the start, participants were requested to speak one at a time so their voice could be recognized for transcription purposes. The focus group followed the topic guide (Appendix 1) and similar pattern to the interviews and was allowed to run its course 
by the interviewees, steered by the moderator to cover all topic areas. The discussion was audio-recorded by two unobtrusive tape recorders.

\section{Data analysis}

The transcripts were examined by two analysts (REEH and $R H$ ) working independently and both using the Framework method (Spencer et al., 2003; Ritchie \& Lewis, 2003) using five distinct stages (Pope \& Mays, 2006): familiarization, development of the thematic framework, indexing, charting and synthesising and finally, mapping and interpretation. On completion of the separate analyses, the findings were reviewed and combined into a single list of three main categories by the lead analyst (REEH). An independent researcher reviewed the transcripts of two of the interviews and one of the focus groups and identified an initial list of themes, which was compared to the themes originally identified. The charts and draft and final themes were then reviewed independently (AMM). A reflexive diary was kept by the lead analyst during the thematic analysis process. This noticed a tendency to dismiss some criticisms of dietitians as the result of unreasonable expectations on the part of the patients. As a result of the diary, the initial list of themes was revised to ensure all data, both positive and negative, was given equal weight within the data indexes and thematic charts. 


\section{Results}

Participants had varying experiences of dietetic consultations. Factors which affected participants' experience are summarised into four main categories: Information, Dietitian's Approach, Dietitian's Behaviour and Appointment (Figure 1). Overall, participants reported a range of experiences, some positive and others negative, and described ways in which their experience of dietetic consultations could have been improved. Direct quotations are used as illustrations and cited using a coded number so that an individual source can be identified with $(P)$ indicating a patient interview and $(\mathrm{F})$, a focus group.

\section{1) Information}

The information and resources that the patient received during their dietetic consultation were an important aspect of their overall experience, and elicited both positive and negative comments.

Resources: Food-wise [the dietitian] has taught me a lot (P1).

Patients identified that receiving information and written resources from the dietitian was an important part of their experience, often helping them to think more about the food they were eating and changing their behaviour. Some patients found that the resources received were 'very beneficial' (P2) or 'helpful' (P3). However, some patients identified written resources as an area for improvement, and noted that information and resources needed to be in a form the patient could understand.

Explanation: [The dietitian] won't just tell me 'Go on, drink orange juice', she explains what [it] does... in the body and why you need to do it (P4). 
Patients valued the fact the dietitian explained the information and advice they gave, and felt that this made them more likely to follow dietary advice and reduced feelings of frustration.

Repetition: A bit later in the conversation [the dietitian] will bring in something we have talked about before (P1).

Patients identified that some information given by the dietitian was repeated each time they attended a consultation. Some patients welcomed this, but others found it 'frustrating' (P5) to have information repeated, if they were not achieving the results they wanted.

Consistency: The second time when I come [sic] there was another dietitian, it was not the first one, and she told me the same thing as well (P4).

Some patients noted dietitians were not the only source of information and advice about their health conditions. They felt it was confusing if they received conflicting information from different sources, and reassuring if more than one health care professional confirmed the same information.

Adaptation: They were...not just sticking to text book answers...[it is important] to adapt this [advice] for personal needs (P6).

Some patients reported dietitians had adapted the advice given to take account of their individual lifestyle. Patients valued the dietitian's recognition of their individual medical, family or economic circumstances and the resulting adaptation of advice. However, some patients felt the advice and information they were given did not take account of their personal circumstances, and therefore it was not relevant to them. 


\section{2) Dietitian's Approach}

Patients identified differences in the overall approach of the dietitian which affected their experience of the consultation.

Prescriptive / Non-Prescriptive: She encourages me to think about it, rather than say 'do this, do that' (P1); [Advice] was a bit liberal, a bit wishy-washy...just say 'well you must do this if you want to lose weight' (P5).

Patients described how some dietitians they had seen used a non-prescriptive approach, guiding rather than instructing and letting the patient set goals and targets. Most of the patients liked this approach. However, other patients wanted a more prescriptive approach, and were not satisfied with the approach taken by their dietitian.

Partnership: ‘a kind of team effort' (P7).

Patients reported that they saw themselves as working together with their dietitian, negotiating action plans and treating the consultation as a partnership. They saw this as an important factor in the effectiveness of the consultation.

Behaviour Change: ...it's about breaking old rules and learning new ones, and it's difficult (F1).

Patients identified that they did not only require factual information to achieve a positive outcome, but were guided and encouraged by the dietitian to make changes to their behaviour, a process which could be 'scary' (P8) and challenging. Patients noted aspects of their behaviour that had changed as a result of seeing their 
dietitian, which increased their feelings of self-esteem, and also identified feelings of guilt associated with not maintaining these changes. Some patients felt that although they were given factual information, they were not given sufficient help in changing their behaviour, and identified this as the reason that they were not achieving the outcomes they wanted: facilitating patients' behaviour changes was seen as key to patients achieving desired outcomes.

Motivation: To have encouragement from the dietitians... even if you've only lost $1 / \mathrm{lb} . . .1 \mathrm{lb}$ is fantastic if you're finding it difficult (P3).

Patients reported that they were more or less motivated by noticing the effect, or lack of effect, of the changes they had made to their behaviour following the advice of a dietitian. Patients felt a dietitian could, in theory, be a source of motivation but, in practice, found some dietitians achieved this whilst others did not; more frequent visits to a dietitian were considered to increase their motivation. Positive feedback from dietitians was important in increasing patients' motivation, and patients felt this feedback should be based on the patient as an individual to maximise its effect.

\section{3) Dietitian's Behaviour}

The way the dietitian behaved during the consultation was important to patients, who identified a number of factors which affected their overall opinion of the consultation.

Listening: [The dietitian] truly listens and wants to know... (P4). She seems interested enough in me as a person/patient to listen to what I am saying (P1).

Patients reported feeling valued when they considered that the dietitian was really listening to them. They considered that a dietitian's use of paraphrasing what they 
had heard from the patient or their reflection of the emotions expressed by the patient were evidence that the dietitian was listening properly. When patients did not feel a dietitian listened, they described the consultation as a negative experience.

Rapport: She certainly had a way about her [which]...made me think that I certainly would have a go [at following advice] (P2).

Patients described a good rapport between themselves and the dietitian as essential. It was important for the dietitian to be approachable and friendly so the patient felt able to share personal information and gained in confidence. Some patients reported experiencing an unpleasant manner in a dietitian, for example 'surly' (P9), and this made it more difficult for them to trust the dietitian.

Body Language: ...her body language is very good for a start ... (P10).

Patients described non-verbal communication as an important aspect of what created a good dietetic consultation. Dietitians' facial expressions (not 'pull[ing] a face' (P1) and 'smiling' (F2) at the patient), 'open body language' (F2) and the placement of the furniture in the consultation room, ('the desk was placed at the side' (F2)), all contributed to patients' overall impression of their experience.

Acceptance: When I come [sic] here at least she listened to me, she, I think she like believed my problems...if you listen to me properly I feel good (P4).

Patients identified that it was important to feel accepted by the dietitian. Patients reported they did not feel judged by their dietitian, and they trusted that the dietitian believed they were speaking truthfully, and that this atmosphere of acceptance made them able to talk more openly. 
Support: The support that was made available to me made all the difference (P7).

Patients felt the dietitian had an important role in providing on-going support as well as factual information and practical advice. Patients felt they often already knew, or were easily provided with all the information they needed but continued support was necessary to achieve their aims. In addition to the dietitian, patients identified peer and group support as important.

Professionalism: I respect her professionality [sic] ... (P10).

The dietitian's status as a professional was important to patients, because it brought with it a 'guaranteed-level' of expertise and standard of behaviour which encouraged patients to value information and advice given. Patients described feeling 'respect' (P10) for their dietitian as a professional.

Empathy: I know you can't order up a whole lot of fat dietitians, but psychologically for the patient if they know you've been through it that helps (P3).

Patients felt it was important for the dietitian to empathize with them, and some doubted a dietitian would be able to do this if $s /$ he had not experienced the same medical condition as the patient. This was particularly true in relation to weight, with one patient describing dietitians as 'beautifully thin' (P3) and suggesting it would be helpful for patients to feel that dietitians could really empathize with them because they had lost weight themselves. This suggests that, in this case, the dietitian was not completely successful in conveying empathy as otherwise her own weight might not have been considered. This example identifies the importance of dietitians identifying and acknowledging this sort of patient feeling. 


\section{4) Appointment}

Patients commented on aspects of their appointment which affected their overall experience of the dietetic consultation but which were often out of the direct control of the dietitian, e.g. availability of appointments, seeing the same dietitian.

Expectation: I went there expecting them to say with a big list .. 'you can't eat this but these are what you can eat' (F1).

Patients described different expectations prior to their first consultation with media representations of nutritionists and experiences in health food stores influencing their understanding of what seeing a dietitian would entail. Some patients were pleased when these expectations were not fulfilled, but others were disappointed by the contrast. Patient expectations of the outcome of their consultations also varied, and they reported feeling frustrated if these expectations (e.g. the expected rate of weight loss) were not met.

\section{Multi-Disciplinary Team:}

Patients felt that dietitians were an important part of the healthcare team. Some patients found a dietitian was more interested in listening and talking through problems than their nurse or general practitioner (GP), but others found other professionals more helpful than a dietitian. Working closely with GPs and other members of the multi-disciplinary team (MDT) was seen as important, and patients found it useful when information regarding their medical condition and treatment was shared between professionals. One patient described dietitians as being on the 
'periphery' (F2) of the MDT, and felt their overall treatment would improve if dietitians worked more closely with other team members.

Regularity/Time: I can go away with all the best intentions and the best pamphlets in the world...if I'm not monitored more closely I will go off the end and just continue to eat what I want (F1).

Appointment frequency varied between patients depending on their medical condition; some patients were satisfied with dietitian availability whereas others felt treatment would be more effective if their appointments were more frequent. Patients felt regular appointments with a dietitian were an important motivation. The length of dietetic consultations also affected patients' experiences. Some patients felt their appointment length was adequate and did not feel restricted by it, whereas others felt the consultation was adversely affected by limited time.

Continuity of Dietitians: ...it would be helpful for us if you're seeing one person ... $(\mathrm{F} 1) ; . .$. doesn't seem to be any continuity, maybe that's why l've had the same thing over and over again .... (P5).

Some patients who had seen a number of different dietitians expressed a preference for continuity and having an appointment with the same practitioner so that the need to repeat introductory discussions was reduced and consultations could progress. Others linked discussion of the continuity of dietitian with the consistency of the information given (see Consistency). Whilst some people expressed a preference for seeing a particular dietitian over another because of their approach, none reported wanting appointments with different dietitians for the sake of variety alone. 
Experience: Within two or three meetings I was feeling much happier... (P10). Well I've done this and l've done this and I'm still putting on weight...you want the dietitian to tell you something that will work (P5).

Patients' overall experience of their consultation varied greatly, and patients described both positive and negative opinions. Patients' views were affected by whether they reported positive outcomes of their dietetic treatment, e.g. weight loss. Patients who had not achieved desired outcomes emphasized wanting effective advice from the dietitian, by which they meant advice that would help them achieve these outcomes. One of the most important factors that impacted on patients' overall experience with a dietitian was whether information they received was 'sufficient' to meet their needs (see Resources) and whether it had been adequately adapted to their individual circumstances (see Adaptation). 'Sufficiency' and adequate adaptation were described as being more important than how often they saw the dietitian. 


\section{Discussion}

The analysis above demonstrates patient experience of dietetic consultations within the small group of patients in the study was not universal, although it was possible to identify common factors that affected experience. Some patients described positive experiences of various aspects of the consultation whilst others described negative experiences of these; some patients were pleased with the outcomes of their dietetic treatment and others were not. Common factors that affected patients' experiences are outlined in the results, but it was not possible to categorize or align a particular type of experience with patients' positive or negative reactions, because different patients reacted to similar experiences with different opinions.

Most of the patients preferred a non-prescriptive, patient-led approach, but some strongly disapproved of this style, stating that they favoured a more prescriptive medical or didactic, practitioner-led style. As discussed above, the patient-centred approach is widely advocated within healthcare, but these results indicate that this term may need redefining as a patient-led approach may not be appropriate for all patients. This finding has been demonstrated by other research: Dowsett et al., (2009), found an overall preference for a patient-led approach in women being treated for breast cancer in Australia but identified a significant minority of patients preferring a 'doctor-centred' style; Swenson et al., (2004), showed a majority of patients in their study in the USA preferred their physician to have a biomedical style approach.

The dietetic patients in this study all emphasized the importance of the dietitian discovering details about them as an individual and tailoring the intervention 
accordingly. In deciding to adopt either a non-prescriptive, patient-led approach or a more prescriptive medical approach, it is important for the dietitian to identify what the patient wants and communicate in the required style, rather than using the same approach for all patients. Pardon et al., (2009), identified a similar need for clinicians to check the information preferences of cancer patients. To be truly patient-centred and to achieve more effective and individually focused care, clinicians may need to decide with each individual patient when this should be patient-led and when this should be practitioner-led, i.e. information given in a medical style, and when to move fluidly between the two within a single consultation. Charles et al., (1999), have described this using the analogy of dancing where the two partners are moving together to music, sometimes with one partner taking the lead and at other times, being led, depending on the beat of the music or the stage of the dance.

The analysis of the patient experiences in the study showed links between positive or negative experience of the dietitian and consultation, and whether or not patients felt that their desired outcomes had been achieved through seeing a dietitian. Patients who had had a negative experience of a dietetic consultation (lack of communication or rapport with dietitian, not given useful information) reported that they had not achieved the outcomes they wanted, and reported that the consultation had not been effective. Patients who were happy with their dietetic consultation also reported being happy with the outcomes. This supports existing research that indicates greater empathy within dietetic consultations improves patient satisfaction (Goodchild et al., 2005). However, whilst acknowledging the importance of empathy, a healthcare professional cannot totally share the patient's lived experience, for example, as described by the patient commenting that 'you can't order up a whole lot 
of fat dietitians' (P3). A dietitian's awareness of the patient's unique expertise in their own experience may help them to optimise their empathic approach and, in turn, to improve the patient's satisfaction with the consultation.

The patients' comments suggest it is crucial for the dietitian to understand the individuality of the patient and to identify the outcome they want from treatment, and to base information and support given on this. Work by MacLellan and Berenbaum (2007), suggests dietitians are aware of this need to identify patient needs and wants, but find it difficult to balance this with the realities of departmental requirements, which may have specific targets or work processes in place, or leave them unable to provide exactly what the patient requires in terms of support or resources. The present study also indicates this issue: patients felt the support provided by the dietitian was sometimes limited by the length or frequency of the appointment or continuity of dietitians, which they recognized was outside of the control of their individual dietitian.

The results suggest there may also be a tension between what the patient wants, and what the dietitian identifies is needed by the patient to improve their health, leaving dietitians uncertain as to how best to practice, which may explain why some patients felt they were given information by the dietitian that related to the dietitian's rather than patient's agenda (MacLellan \& Berenbaum, 2007). Overlooking the patients' wants in favour of what the dietitian/expert identifies as suitable information creates a situation in which the patient feels the need to rationalize their behaviour and reject suggestions for change (Hunt \& Pearson, 2001), which is perhaps why some patients felt advice they had been given was impossible for them to implement. 
This study identifies the importance of the dietitian communicating well and building a rapport with patients, providing good quality information which the patient can rely on and which meets the patient's needs as well as continuity of care and providing individualized understanding, acceptance and emotional support. This concurs with other studies, which have identified the importance to patients of 'being taken seriously' and listened to in a sensitive way (Kerssens \& van Yperen, 1996), the value of communication skills (Cant \& Aroni, 2008; Cant, 2009), developing a relationship by seeing the same practitioner (Ridd et al., 2009) and the value of the dietitian in providing emotional support (Jones et al., 2007). Some patients also noted the value of support from peers with similar medical conditions, and this concurs with experience of other patients who have noted the value of group support and 'expert patient' assistance (Hollingdale et al., 2008).

Patients identified frequent appointments as an important motivating factor in achieving behaviour change. This concurs with work looking at weight management patients who are more successful in achieving weight loss with regular support (Douketis et al., 1999; Uusitupa et al., 2000; Munnelly \& Feehan, 2002). Availability of appointments is not always within the control of the individual dietitian, but acknowledging the importance of frequent support and facilitating this whenever possible may help patients to maintain their level of motivation and achieve the treatment outcomes they want.

The limitations of this study include the small sample size which reflects the qualitative nature of this investigation. The method of participant recruitment was not 
based on systematically random sampling so the findings cannot be considered representative of a wider population nor used as the basis of numerical extrapolation. The approach to the overall study and analysis was considered rigorous (Ritchie \& Lewis, 2003) but the research team comprised only dietitians and student dietitians. Whilst they endeavoured to minimize professional bias and to be as objective as possible, for example by the use of a reflexive diary kept by the lead analyst, it could be argued that the presence of a non-dietitian on the research team may have enhanced impartiality.

The findings from this study could be used to developed a questionnaire for use in a larger quantitative investigation to explore the relative proportions of patients with preferences for different approaches in dietetic consultations and any potential relationships with demographic or health variables. Future intervention studies could then be used to explore the value of targeted approaches in order to enhance patients' experience and improve outcome measures.

In conclusion, this study shows that patients' experiences are varied and their perceptions and judgments of consultations are highly individual. These experiences are affected by many factors which have been broadly categorized above. In order for the dietitian to ensure that the patient has a positive and effective experience of the consultation, they must identify information about the patient as an individual, and fully understand the patient's expectations, goals and needs. This patientcentred approach may need to be either patient-led or practitioner-led and dietitians should, therefore, be flexible and responsive in their approach to ensure that they meet each patient's needs. 


\section{Acknowledgements}

The authors would like to thank (1) The Health Foundation who provided financial support for Gillie Bonner through a 'Leading Practice Through Research Award'; (2) the patients and dietitians who participated in the study; (3) Stephanie Turner who provided an independent review of a sample of the study transcripts. 


\section{References}

Audit Commission (1993) What seems to be the matter? Communication between hospitals and patients. London: HMSO.

Boffetta, P., Couto, E., Wichmann, J., Ferrari, P., Trichopoulos, D., Bueno-deMesquita, H.B., van Duijnhoven, F.J.B., Buchner, F.L., Key, T., Boeing, H. et al. (2010) Fruit and vegetable intake and overall cancer risk in the European Prospective Investigation into Cancer and Nutrition (EPIC). J. Natl. Cancer Inst. 102, 529-37.

Bonner, G., Madden, A.M., Baker, A. \& Jones, J.M. (2008) The validation of a rating scale to assess dietitians' use of behaviour change skills. J. Hum. Nutr. Diet. 21, 381.

Cant, R.P. (2009) Communication competence within dietetics: dietitians' and clients' views about the unspoken dialogue - the impact of personal presentation. J. Hum. Nutr. Diet. 22, 504-10.

Cant, R.P. \& Aroni, R.A (2008) Exploring dietitians' verbal and non-verbal communication skills for effective dietitian-patient communication. J. Hum. Nutr. Diet. 21. 502-11.

Carter, S.J., Roberts, M.B., Salter, J. \& Eaton, C.B. (2010) Relationship between Mediterranean diet score and atherothrombotic risk: Findings from the third National 
Health and Nutrition Examination Survey (NHANES III), 1988-1994. Atherosclerosis 210, 630-6.

Charles, C., Gafni, A. \& Whelan, T (1999) Decision-making in the physician-patient encounter: revisiting the shared treatment decision-making model. Soc. Sci. Med. $49,651-61$.

Curry, K.R. \& Jaffe, A. (1998) Nutrition counselling and communication skills. Philadelphia: Saunders.

Department of Health (2010). The handbook to the NHS constitution for England. http://www.dh.gov.uk/en/Publicationsandstatistics/Publications/PublicationsPolicyAn dGuidance/DH 113613 accessed 27 October 2011.

Dietitians of Canada (1996). Competencies for the entry-level dietitian. http://www.dietitians.ca/Downloadable-Content/Public/Competencies for Entrylevel Dietitian.aspx accessed 27 October 2011.

Douketis, J.D., Feightner, J.W., Attia, J. \& Feldman, W. (1999) Periodic health examination 1999, update: 1. detection, prevention and treatment of obesity. Can. Med. Assoc. J. 160, 513-25.

Dowsett, S.M., Saul, J.L., Butow, P.N., Dunn, S.M., Boyer, M.J., Findlow, R. \& Dunsmore, J. (2000) Communication styles in the cancer consultation: preferences for a patient-centred approach. Psycho-Oncology 9, 147-56. 
Ferguson, M., Capra, S., Bauer, J. \& Banks, M. (2001) Development of a patient satisfaction survey with inpatient clinical nutrition services. Aust. J. Nutr. Diet. 58, $157-63$.

Gable, J. (2007) Counselling skills for dietitians, $2^{\text {nd }}$ edn. Oxford: Blackwell.

Goodchild, C.E., Skinner, T.C. \& Parkin, T. (2005) The value of empathy in dietetic consultations: a pilot study to investigate its effect on satisfaction, autonomy and agreement. J. Hum. Nutr. Diet. 18, 181-5.

Goodpaster, B.H., DeLany, J.P., Otto, A.D., Kuller, L., Vockley, J., South-Paul, J.E., Thomas, S.B., Brown, J., McTigue, K., Hames, K.C., Lang, W. \& Jakicic, J.M. (2010) Effects of diet and physical activity interventions on weight loss and cardiometabolic risk factors in severely obese adults. J. Amer. Med. Assoc. 304, 1795-802.

Health Professions Council (2007) Standards of proficiency. http://www.hpcuk.org/assets/documents/1000050CStandards of Proficiency Dietitians.pdf accessed 27 October 2011.

Hollingdale, R., Sutton, D. \& Hart, K. (2008) Facilitating dietary change in renal disease: investigating patient's perspectives. J. Ren. Care 34, 136-42.

Hunt, P. \& Pearson, D. (2001) Motivating Change. Nurs. Stand 16, 45-52. 
Jones, N., Furlanetto, D.L., Jackson, J.A. \& Kinn, S. (2007) Investigation into obese adults' views of the outcomes of dietary treatment. J. Hum. Nutr. Diet. 20, 486-94

Kerssens, J.J. \& van Yperen, E.M. (1996) Patients' evaluation of dietetic care: testing a cognitive attitude approach. Patient Educ. Couns. 27, 217-226.

Lewin, S., Skea, Z., Entwistle, V.A., Zwarenstein, M. \& Dick, J. (2001) Interventions for providers to promote a patient-centred approach in clinical consultations. Cochrane Database of Systematic Reviews, Issue 4. Art. No.: CD003267. DOI: 10.1002/14651858.CD003267.

Lu, A.H. \& Dollahite, J. (2010) Assessment of dietitians' nutrition counselling selfefficacy and its positive relationship to reported skill usage. J. Hum. Nutr. Diet. 23, $144-53$.

MacLellan, D.L. \& Berenbaum, S. (2006) Dietitians' opinions and experiences of client centred nutrition counselling. Can. J. Diet. Prac. Res. 67, 119-24.

MacLellan, D. \& Berenbaum, S. (2007) Canadian dietitians' understanding of the client-centered approach to nutrition counselling. J. Am. Diet. Assoc. 107, 1414-7.

Mead, N. \& Bower, P. (2000) Patient-centredness: a conceptual framework and review of the empirical literature. Soc. Sci. Med. 51, 1087-110.

Munnelly, P. \& Feehan, S. (2002) An obesity clinic model. Proc. Nutr. Soc. 61, 9-10. 
Najavits, L.M. \& Weiss, R.D. (1994) Variations in therapist effectiveness in the treatment of patients with substance use disorders: an empirical review. Addiction 89, 679-88.

O'Sullivan-Maillet, J., Skates, J. \& Pritchett, E. (2005) American Dietetic Association: Scope of dietetics practice framework. J. Am. Diet. Assoc. 105, 634-40.

Pardon, K., Deschepper, R., Stichele, R.V., Bernheim, J., Mortier, F. \& Deliens, L. (2009) Preferences of advance lung cancer patients for patient centred information and decision-making: A prospective multi-centred study in 13 hospitals in Belgium. Patient Educ. Couns. 77, 421-9.

Pope, C. \& Mays, N. (2006) Qualitative research in healthcare, $3^{\text {rd }}$ edn. London: Wiley-Blackwell (BMJ Books).

Ridd, M., Shaw, A., Lewis, G. \& Salisbury, C. (2009) The patient-doctor relationship: A synthesis of the qualitative literature on patients' perspectives. Br. J. Gen. Pract. 59, e116-133.

Ritchie, J. \& Lewis, J. (2003) Qualitative research practice: A guide for social science students and researchers. London: Sage. 
Spencer, L., Ritchie, J., Lewis, J. \& Dillon, L. (2003) Quality in qualitative evaluation: A framework for assessing research evidence. London: Government Chief Social Researcher's Office.

Swenson, S.L., Buell, S., Zettler, P., White, M., Ruston, DC. \& Lo, B. (2004) Patientcentered communication: do patients really prefer it? J. Gen. Intern. Med. 19, 106979.

Stewart, M. \& Browne, J.B. (1996) Patient centredness in medicine. In: Evidencebased patient choice, ed T Hope, pp 19-28. London: The King's Fund.

Uusitupa, M., Louheranta, A., Lindstrom, J., Valle, T., Sundvall, J. \& Tuomilehto, J. (2000) The Finnish diabetes prevention study. Br. J. Nutr. 83 (Suppl. 1), S137S142.

Vivanti, A., Ash, S. \& Hulcombe, J. (2007) Validation of a satisfaction survey for rural and urban outpatient dietetic services. J. Hum. Nutr. Diet. 20, 41-9.

Whitehead, K., Langley-Evans, S.C., Tischler, V. \& Swift, J.A. (2009) Communication skills for behaviour change in dietetic consultations. J. Hum. Nutr. Diet. 22, 493-500.

World Health Organization (2000) The world health report 2000 - health systems: Improving performance. Geneva: World Health Organization http://www.who.int/whr/2000/en/whr00 en.pdf accessed 27 October 2011. 


\section{APPENDIX 1 - Interview and focus group topic guide}

Key objective: To elucidate any links between dietitians' practices and patient responses in terms of perceived autonomy support, motivation, self-efficacy and overall satisfaction with consultation.

1. As participants arrive welcome them individually, provide refreshments, and obtain consent.

2. Start proceedings by introducing self.

3. Introduce the research (background, organization + funding), purpose of the group, plans for data analysis and reporting.

4. Establish ground rules (confidentiality, all viewpoints welcomed, speak one at a time etc)

5. Invite each participant to introduce him/herself.

6. Introductory topics:

- Overall feelings about your consultation;

- Overall impact on your diet/eating behaviour.

7. Main body topics:

Review what dietitians did/said that resulted in participants feeling (or feeling the opposite of):

- Satisfied with the consultation

- Motivated to make/sustain changes to their diet

- Confident about making/sustaining changes to their diet

- Accepted and understood Use prompts/vignettes around things the dietitian may have done e.g.

- Listens attentively

- Seems genuinely interested in helping

- Helps you to work things out for yourself (e.g. problem solving, goal setting, pros and cons, information provision)

- Helps you to think about things differently/see things from another point of view

- Helps you to plan how to cope with things that might get in the way of your healthy eating goals

8. (if time) Draw out additional topics that have arisen (e.g. things other than dietitians' practice that impacted outcome)

9. Move discussion towards ending:

- Consider how participants would like dietitians to help, what they would like to see come out of this research.

10. Concluding remarks and thank-you.

11. Arrange remuneration for expenses. 


\section{APPENDIX 2 - Initial conceptual framework index}

1. Patient Expectation

1.1. Expectation of What Consultation Would Be

1.2. Expectation of Advice Received

2. Patient Experience

2.1. Happy with Consultation in General

2.2. Unhappy with Consultation in General

3. Patient Opinion of Dietitian

3.1. Feeling of Acceptance

3.2. Empathy/Understanding/Support

3.3. Listening/Communication

3.4. Judgmental/Non-Judgmental

3.5. Dietitian's Manner/ Rapport

3.6. Dietitian's Experience/Weight

3.7. Dietitian's Body Language

4. Information Received

4.1. Resources

4.2. Explanation of Advice Given

4.3. Prescriptive Approach

4.4. Non-Prescriptive/Collaborative Approach

4.5. Patient's Opinion of Information Given

4.6. Conflicting Information

4.7. Group/Peer Support

5. NHS/System Experience

5.1. Time

5.2. Access to Dietitian

5.3. Other Health Care Professionals

5.4. Varying Dietitians Seen

6. Patient As Individual

6.1. Level of Personal Information Discussed During Consultation

6.2. Adaptation of Advice for Individual

6.3. Holistic factors

6.4. Family Impact

6.5. Information the patient Wants to Know

7. Result of Consultation

7.1. Patient felt In Control

7.2. Satisfaction

7.3. Behaviour Change

7.4. Motivation

8. Patients' Internal Experience

8.1. Guilt

8.2. Sadness 


\subsection{Frustration \\ 8.4. Impatience}

9. Other Issues

9.1. Background: Reason for Dietetic Referral, Times Dietitian Seen

9.2. Information From Non-Health Professional Sources

9.3. Other Issues Not Covered Above 
Figure 1

Factors affecting participants' experience of dietetic consultations
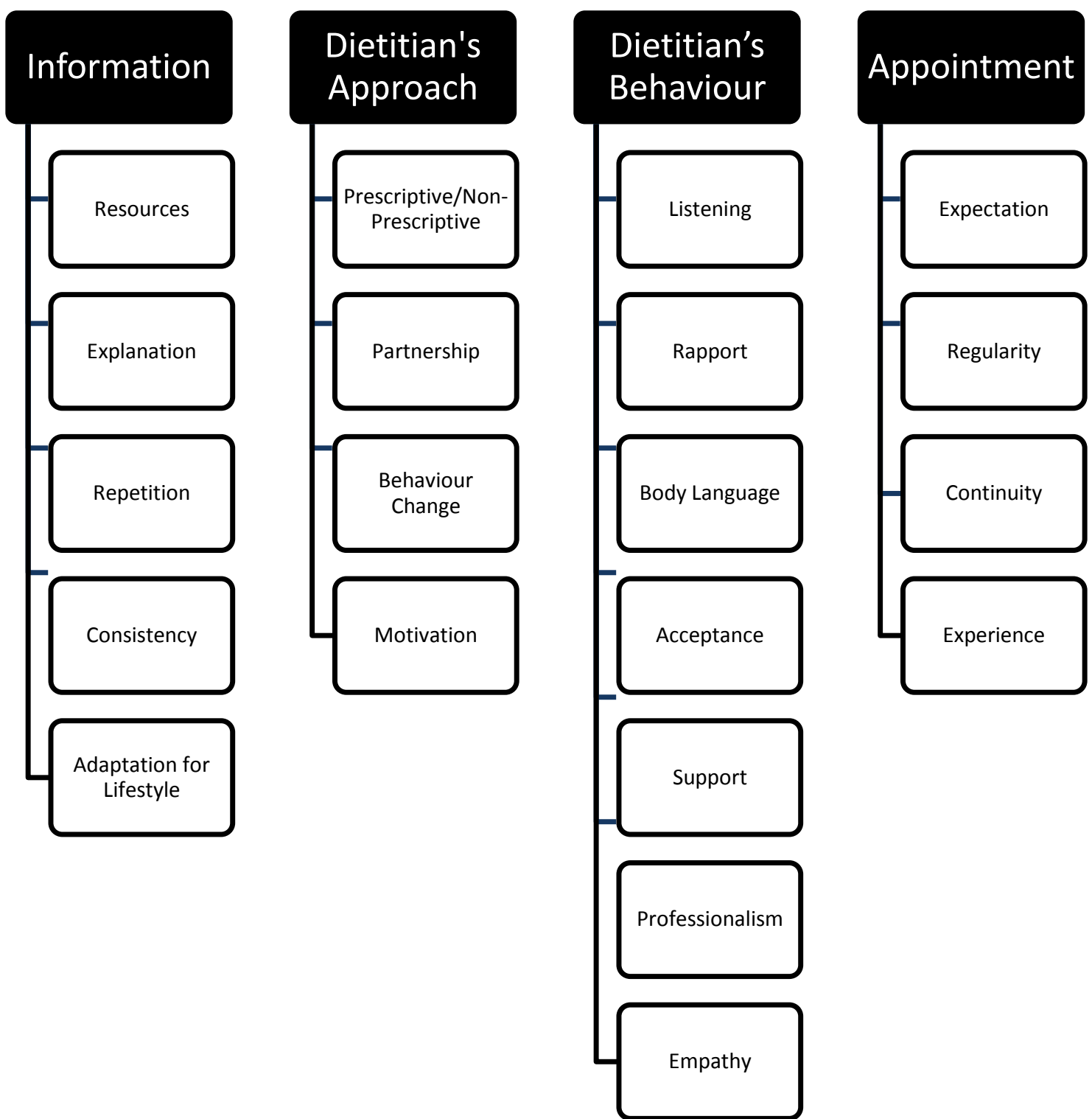